\title{
Effect of physical exercise on cognitive function in older adults' carriers versus noncarriers of apolipoprotein E4: systematic review and meta-analysis
}

\author{
José María Cancela-Carral ${ }^{1,2,3 *}$, Adriana López-Rodríguez ${ }^{2,3}$, Irimia Mollinedo-Cardalda ${ }^{2,4}$ \\ ${ }^{1}$ HealthyFit Research Group, University of Vigo, Pontevedra, Spain \\ ${ }^{2}$ Galicia Sur Health Research Institute (IIS Galicia Sur), Sergas-UVIGO, Pontevedra, Spain \\ ${ }^{3}$ Faculty of Education and Sports Science, University of Vigo, Pontevedra, Spain \\ ${ }^{4}$ Faculty of Physiotherapy, University of Vigo, Pontevedra, Spain
}

The presence of apolipoprotein (Apo) E4 is a genetic risk factor in cognitive impairment. Physical exercise contributes to slowing cognitive impairment in older adults, but little is known about the influence of exercise on ApoE4 carriers and noncarriers. The objective of systematic review is to study the role of physical exercise in older adults' ApoE4 carriers and noncarriers. A systematic literature search was carried out in five international databases: PubMed, Web of Science, PeDro, Scopus, and SPORTDiscus. A total of nine randomized controlled trials were included with a sample size of 2,025 subjects ( 901 ApoE4 carriers). The exercise reported a significant improvement on cognitive performance in older adults' ApoE4 noncarriers (standardized mean difference $[\mathrm{SMD}]=0.653 ; 95 \%$ confidence interval $[\mathrm{Cl}], 0.29-1.00 ; \mathrm{chi}^{2}=35.36$; degrees of freedom $[d f]=7 ; P<0.0001 ; I^{2}=80 \%$ ). It was also reported that a total program duration greater than 50 sessions generated different and significant effects on cognitive performance in older adults' ApoE4 noncarriers (SMD =0.878; 95\% Cl, 0.14-1.61; $\operatorname{chi}^{2}=31.82 ; d f=3$; $\left.P<0.0001 ; P^{2}=91 \%\right)$. The results reported that high intensity generated a differential effect on cognitive performance in older adults' ApoE4 carriers versus noncarriers $\left(S M D=0.963 ; 95 \% \mathrm{Cl}, 0.25-1.67 ; \mathrm{chi}^{2}=18.11 ; d f=3\right.$; $\left.P<0.0004 ; I^{2}=83 \%\right)$. The effect of physical exercise on cognitive performance in older adults is conditioned by the presence or not of ApoE4.

Keywords: Apolipoproteins E, Exercise, Cognition, Aged

\section{INTRODUCTION}

The population aged 60 and over will be 1.4 trillion by 2030 , estimated that by 2050 the global elderly population will be close to 2.1 trillion people (United Nations, 2015). The increase in life expectancy and consequently the increase in the ageing population in accompanied by an increase in the prevalence of age-related diseases, such as dementia or cognitive impairment. Dementia is defined as a disorder characterized by cognitive decline acquired enough severity to affect social and/or professional functioning (Hugo and Ganguli, 2014). Mild cognitive impairment is common condition in older age and is associated with an increased risk of future cognitive decline and dementia (Jongsiriyanyong and Limpawattana, 2018; Lautenschlager et al., 2019) and involves cognitive alterations that, without meeting dementia criteria or significantly affecting functional capacity, represents a deterioration from what is expected by age, and identifies a group of people at risk of developing dementia in the future (Lautenschlager et al., 2019). Dementia has complex genetics and is associated with both genetic and environmental factors. In addition to age, which is an important risk factor for the development of dementia (Amieva et al., 2004), the main genetic risk factor for cognitive decline and dementia is the presence of apolipoprotein (Apo) E4 (Rawle et al., 2018).

$A p o E$ is a gene responsible for encoding a protein that circulates in plasma and is present in the central nervous system, helping to
*Corresponding author: José María Cancela-Carral

(D) https://orcid.org/0000-0003-2903-3829

HealthyFit Research Group, Faculty of Education and Sports Science, University of Vigo, 36005 Campus A Xunqueira s/n, Pontevedra, Spain

Email: chemacc@uvigo.es

Received: February 19, 2021 / Accepted: March 13, 2021
This is an Open Access article distributed under the terms of the Creative Commons Attribution Non-Commercial License (https://creativecommons.org/licenses/by-nc/4.0/) which permits unrestricted non-commercial use, distribution, and reproduction in any medium, provided the original work is properly cited. 
regulate cholesterol, lipid metabolism, cell repair processes (Huebbe et al., 2011; Mahley and Huang, 2012) and eliminate A-1-42 from the brain (Yu et al., 2014). In addition, intracellular $A p o E$ modulates cytoskeleton stability processes, mitochondrial integrity, and dendrite morphology (Huang and Mahley, 2014). The ApoE gene is located on chromosome 19, is polyphormic and has three main isoforms: $A p o E 2, A p o E 3$, and ApoE4 (Corbo and Scacchi, 1999). The ApoE2 and $A p o E 3$ alleles confer reduced risks of ageing-related diseases, however, people carrying the ApoE4 allele increase the risk of Alzheimer disease (AD) (Michaelson, 2014; Rajmohan and Reddy, 2017; Tai et al., 2016), 3 times while carrying two copies of the allele $\varepsilon 4$ can increase it up to 15 times (Qian et al., 2017).

Physical exercise may thus contribute to preventing and/or delaying $\mathrm{AD}$, cognitive decline, and other types of dementia in older adults (Stephen et al., 2017) even in carriers of the ApoE4 allele. The current literature includes several studies investigating the protective effect of physical exercise on older adults' ApoE4 carriers, reducing the risk of cognitive decline compared to sedentary people (Smith et al., 2011; Smith et al., 2013; Woodard et al., 2012). Based on scientific evidence, we can indicate that physical exercise can significantly reduce the risk of coronary artery disease and improve cognitive aging and EA biomarkers in ApoE4 carriers (Erickson et al., 2012).

Currently, there is already important research that analyses the influence that physical exercise has to slow the onset of cognitive decline and Alzheimer disease in the population carrying ApoE4. Defining optimal preventive guidelines in terms of kind, duration (length of whole intervention) and intensity of physical exercise intervention program is a very important issue. This systematic review and meta-analysis study the role of physical exercise as a potentially preventive intervention against cognitive decline in older adults' ApoE4 carries and noncarriers, by evaluating the quality of available evidence. It also aims to investigate what type of physical exercise, how much (number of weeks of intervention) and at what intensity generates a differential effect on cognitive performance in older adults' ApoE4 carries and noncarriers.

\section{MATERIALS AND METHODS}

This systematic review and meta-analysis were registered in a publicly accessible registry, the PROSPERO database (registration number: CRD42020211672). Study report followed the Preferred Reporting Items for Systematic Reviews and Meta-Analyses (PRISMA) guidelines (Moher et al., 2009).

\section{Search strategy}

A systematic literature search was performed for eligible studies published before December 2020 on five international databases (PubMed, Web of Science, PeDro, Scopus, and SPORTDiscus). Key words as "physical activity" OR "exercise" AND "ApoE4" OR “Apolipoprotein E4” AND "cognition." Thirty-six studies were retrieved in this search, which were supplemented by manual searches through reference lists of published reports. Titles and abstracts from a final total of 18 studies were then reviewed for further inclusion. All analyses were based on published studies; therefore, no ethical approval and patient consent are required.

\section{Criteria for inclusion}

Studies were included that met the following inclusion criteria: (a) study population must be composed of people 60 years or over (de Souto Barreto et al., 2018); (b) included subjects ApoE4 carries and noncarriers (Schuit et al., 2001); (c) involved an exerciseonly intervention in the experimental group under the guarantee of basically medical care (Jia et al., 2019); (d) studies that are randomized controlled trial (RCT). Two authors independently reviewed full text of all articles that were considered relevant for inclusion in this review. A third author was consulted in cases of disagreements between these two authors. The study selection process is described in Fig. 1.

\section{Criteria for exclusion}

Studies that clearly did not meet initial criteria were rejected on initial review. Reviews, conference papers, abstracts without available full text and studies written in languages other than English or Spanish were also excluded. Studies where the size of the experimental group was not equal to or greater than 15 were also excluded.

\section{Data extraction}

Titles and abstract of studies identified in the initial search were imported into Refworks (reference management software). After duplicates, studies that are clearly irrelevant to the effects of physical activity in the older adults with ApoE4 were removed; full text of potentially relevant papers was imported for further screening. Two authors performed an initial screening of titles and abstracts independently followed by a screening potential relevant full text guided by inclusion criteria. A third reviewer was consulted for any screening disagreement. From each study, we collected the following data if available: year of publication, sample size, participants' characteristics (age, number of participants from 


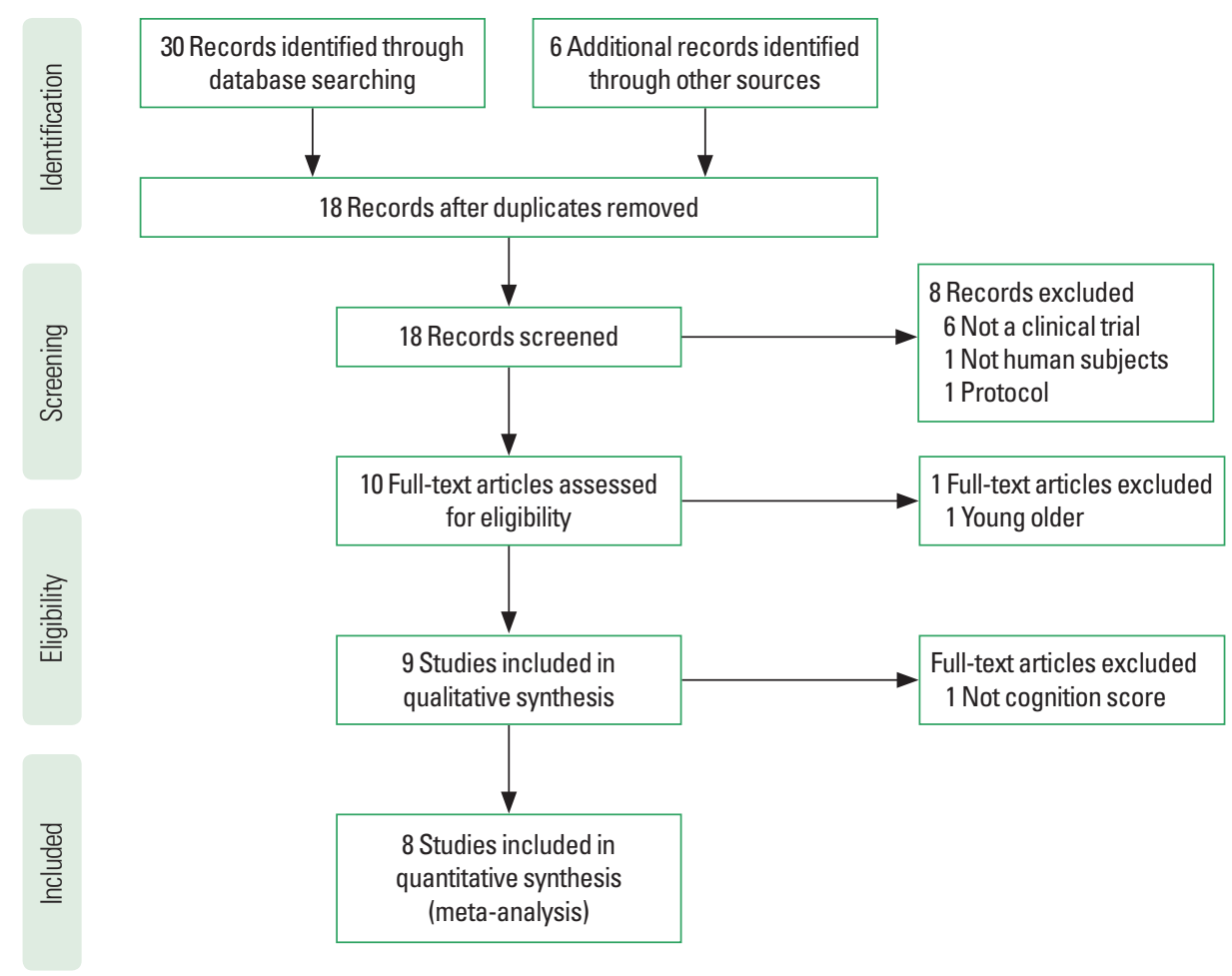

Fig. 1. Study selection process.

experimental groups and control group), intervention characteristics (type of intervention, type of exercise, length of whole duration, frequency, intensity, progression, place, supervision, adherence, dropouts during intervention) and consensus on exercise reporting template (CERT), measurement tools, limitations, and findings. Relevant information was extracted by two reviewers independently onto a standard template.

\section{Risk of bias}

Studies meeting inclusion criteria were individually scored by two authors independently according to the Cochrane risk of bias tool, the third author would be consulted when disagreement appeared. Seven items regarding risk of bias was assessed: random sequence generation, allocation concealment, blinding of participants and personnel, blinding of outcome assessment, incomplete outcome data, selective outcome reporting, and other sources of bias. The included RCT's were classified as being at low risk, high risk, or unclear in the above fields.

\section{Data analysis}

Meta-analysis was conducted using Review Manager Software (RevMan 5.3). The continuous outcome such as cognitive perfor- mance, the standardized mean difference (SMD) was calculated. All differences calculated were expressed as $95 \%$ confidence intervals (CI). All data analyses were performed with the IBM SPSS Statistics ver. 25.0 (IBM Co., Armonk, NY, USA). Due to heterogeneity between studies, a random-effects model was used for this meta-analysis. Subgroup analyses were conducted to explore the differences among the effects of duration and intensity of exercise program on cognitive performance of subjects.

\section{Meta-analysis}

Random-effects meta-analysis was selected due to the existence of methodological heterogeneity across studies. SMDs were pooled because outcomes were continuous. Heterogeneity across studies was quantified using $I^{2}$ statistic (variation in SMD attributable to heterogeneity).

\section{Subgroup analysis}

Studies were grouped based duration (length of whole duration) and intensity of exercise intervention program. Intervention effects were estimated within subgroups and compared across subgroups to identify components that modify the intervention effects. 


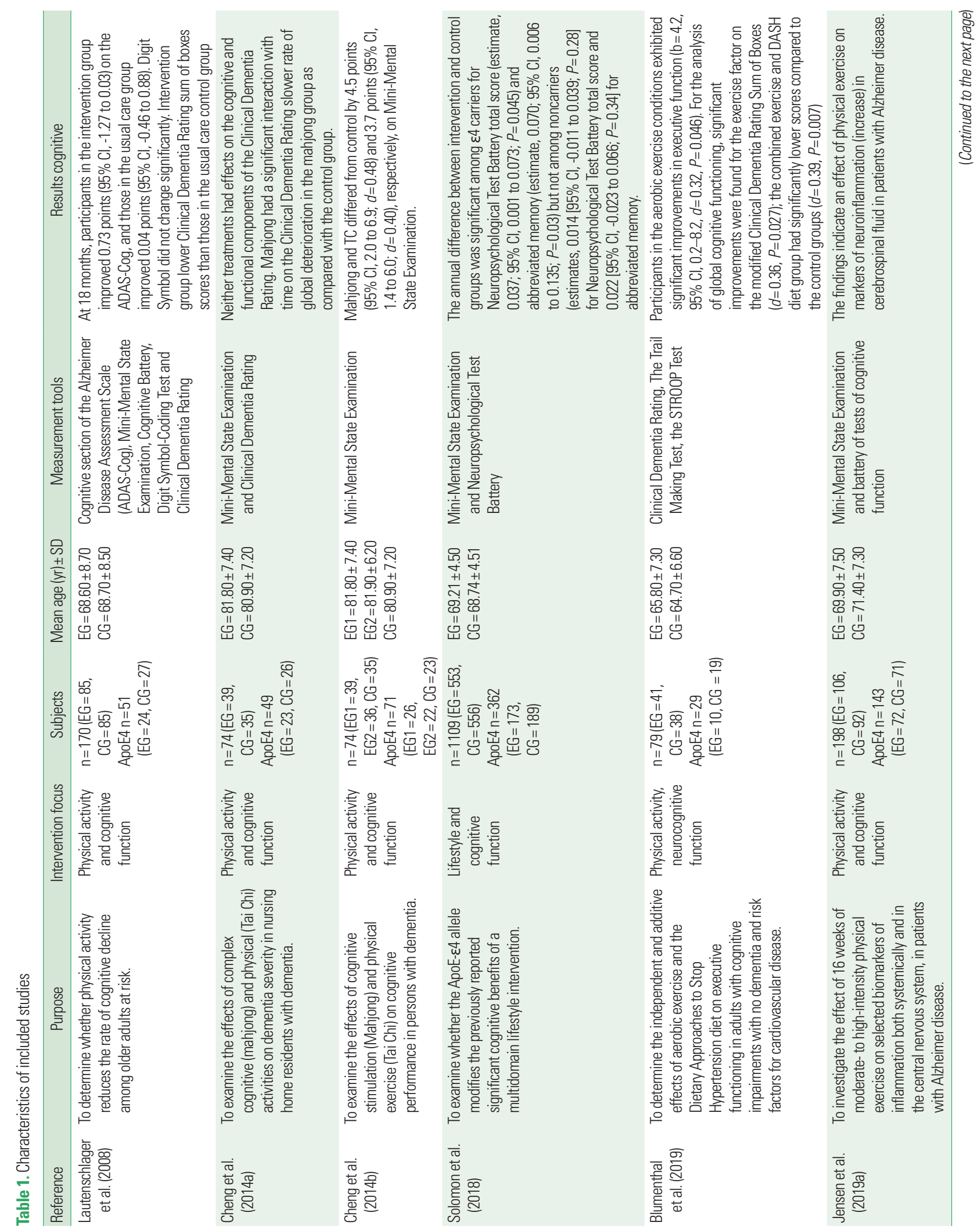




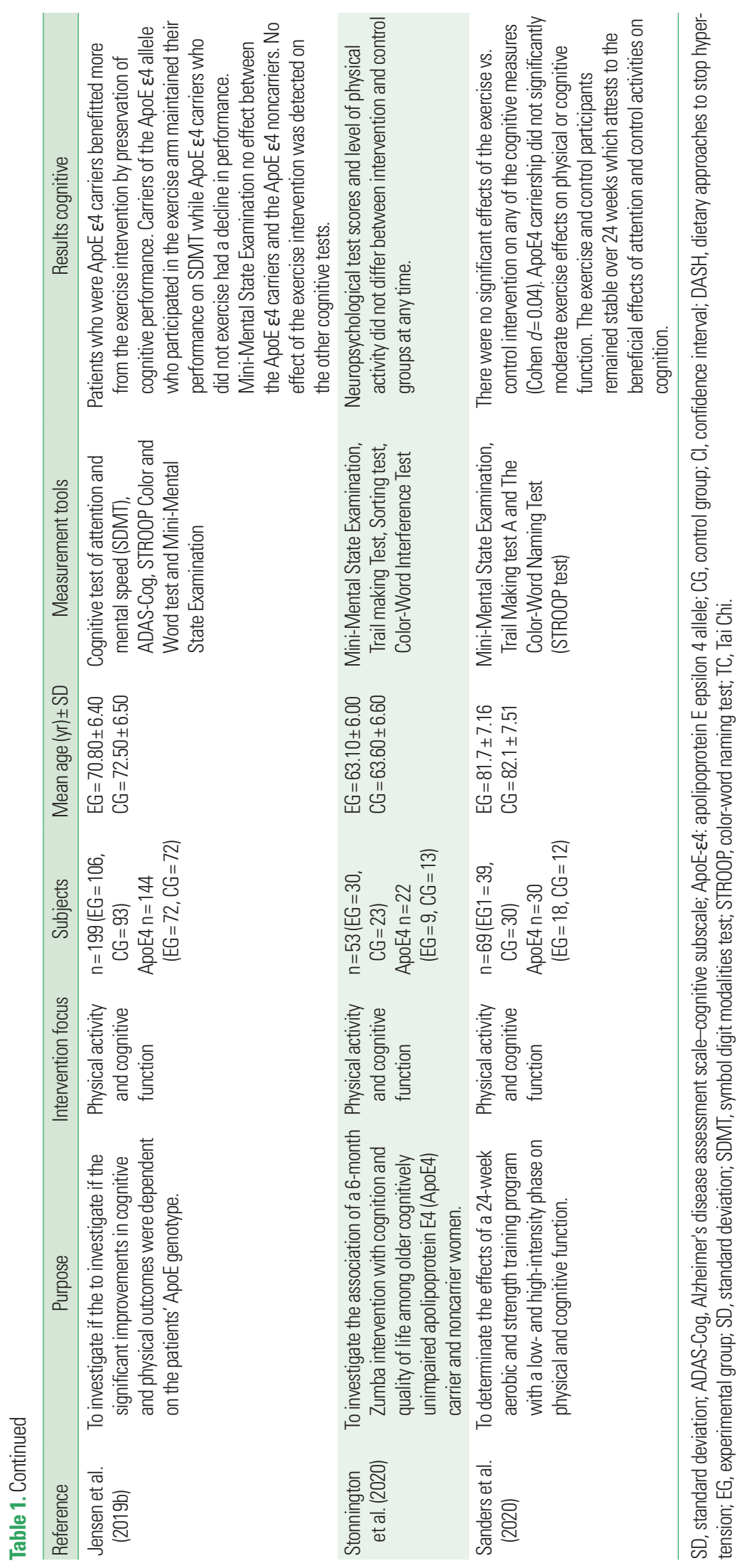




\section{RESULTS}

\section{Study selection}

The original search identified 36 citations underwent a full-text screen by two independent reviewers, after duplicates, studies irrelevant to $\mathrm{AD}$ and exercise or physical activity, animal trails and reviews were removed. A total of nine trials were included in the final review. The meta-analysis was developed with eight trials, because a trial did not provide sufficient quantitative cognitive performance information. A full report of the selection process can be found in the PRISMA diagram in Fig. 1.

\section{Description of studies}

Descriptive characteristics for nine included studies are presented in Table 1. In total, the trials included 2,025 participants of which 901 will be ApoE4 carries. The nine included studies were published between 2008 and 2020. All studies reported beneficial effects of physical activity on cognition of patients. Table 2 presented the degree of risk of bias for all studies included. The random assignment procedure were reported in nine studies (Blumenthal et al., 2019; Cheng et al., 2014a; Cheng et al., 2014b; Jensen et al., 2019a; Jensen et al., 2019b; Lautenschlager et al., 2008; Sanders et al., 2020; Solomon et al., 2018; Stonnington et al., 2020) only two studies reported a blinding procedure for participants and personnel (Blumenthal et al., 2019; Solomon et al., 2018). Blinding of outcome assessment was presented in four studies (Lautenschlager et al., 2008; Sanders et al., 2020; Solomon et al., 2018; Stonnington et al., 2020). All included trials incomplete outcome data and selective reporting (Blumenthal et al., 2019; Cheng et al., 2014a; Cheng et al., 2014b; Jensen et al., 2019a; Jensen et al., 2019b; Lautenschlager et al., 2008; Sanders et al., 2020; Solomon et al., 2018; Stonnington et al., 2020).

\section{Participants' characteristics}

All included trials contained a sample size of 2,025 subjects. Exercise and control groups were composed of 1,038 and 987 subjects respectively. From 2,025 subjects 901 will be ApoE4 carriers. Exercise and control groups were composed of 449 and 452 subjects respectively. Subjects' average age was 73.06 \pm 7.11 (Table 1).

\section{Intervention characteristics}

Table 3 showed that of nine included studies, only two articles (Cheng et al., 2014a; Cheng et al., 2014b) do not report information on the intensity of the exercises, all the others are between moderate or high intensity carried out moderate- to high-intensity intervention, the rest of included studies conducted. Aerobic exercise was chosen by seven studies (Blumenthal et al., 2019; Cheng et al., 2014a; Cheng et al., 2014b; Jensen et al., 2019a; Jensen et al., 2019b; Lautenschlager et al., 2008; Stonnington et al., 2020), and two of those seven studies combined aerobics with strength (Sanders et al., 2020; Solomon et al., 2018). All interventions except in one study (Solomon et al., 2018) that does not report information were conducted at an average of $52.14 \pm 11.49 \mathrm{~min}$ a session ranging from 30 (Sanders et al., 2020) to $60 \mathrm{~min}$ (Cheng et al., 2014a; Cheng et al., 2014b, Jensen et al., 2019a; Jensen et al., 2019b; Stonnington et al., 2020). Interventions were conducted at least 2 days for week. Length of whole duration ranges from 12 to 96 weeks, mean intervention duration was $33.33 \pm 30.65$ weeks. In terms of adherence to interventions in the studies varied between 60\% (Sanders et al., 2020) and 100\% (Cheng et al., 2014a),

Table 2. Assessment for the risk of bias in included studies

\begin{tabular}{|c|c|c|c|c|c|c|c|c|}
\hline \multirow[b]{2}{*}{ Reference } & \multicolumn{2}{|c|}{ Selection bias } & \multirow{2}{*}{$\begin{array}{c}\text { Performance bias } \\
\text { Blinding of } \\
\text { participants and } \\
\text { personnel }\end{array}$} & \multirow{2}{*}{$\begin{array}{l}\text { Detection bias } \\
\text { Blinding of } \\
\text { outcome } \\
\text { assessment }\end{array}$} & \multirow{2}{*}{$\begin{array}{c}\text { Attrition bias } \\
\text { Incomplete } \\
\text { outcome data }\end{array}$} & \multirow{2}{*}{$\begin{array}{c}\text { Reporting bias } \\
\begin{array}{c}\text { Selective } \\
\text { reporting }\end{array}\end{array}$} & \multirow[b]{2}{*}{$\begin{array}{l}\text { Other } \\
\text { sources } \\
\text { of bias }\end{array}$} & \multirow[b]{2}{*}{ Tota } \\
\hline & $\begin{array}{c}\text { Random } \\
\text { sequence } \\
\text { generation }\end{array}$ & $\begin{array}{l}\text { Allocation } \\
\text { concealment }\end{array}$ & & & & & & \\
\hline Lautenschlager et al. (2008) & (1) & 2 & (2) & (1) & (1) & (1) & 0 & 8 \\
\hline Cheng et al. (2014a) & (1) & (2) & (2) & (2) & (1) & (1) & 0 & 9 \\
\hline Cheng et al. (2014b) & (1) & (2) & (2) & (2) & (1) & (1) & 0 & 9 \\
\hline Solomon et al. (2018) & (1) & (1) & (1) & (1) & (1) & (1) & 0 & 6 \\
\hline Blumenthal et al. (2019) & (1) & (1) & (1) & (2) & (1) & (1) & 0 & 7 \\
\hline Jensen et al. (2019a) & (1) & 0 & 0 & 0 & (1) & (1) & 0 & 3 \\
\hline Jensen et al. (2019b) & (1) & 0 & 0 & 0 & (1) & (1) & 0 & 3 \\
\hline Stonnington et al. (2020) & (1) & 0 & 0 & (1) & (1) & (1) & 0 & 4 \\
\hline Sanders et al. (2020) & (1) & 0 & 0 & (1) & (1) & (1) & 0 & 4 \\
\hline
\end{tabular}

1, low risk of bias; 2 , high risk of bias; 0 , unclear risk of bias. 


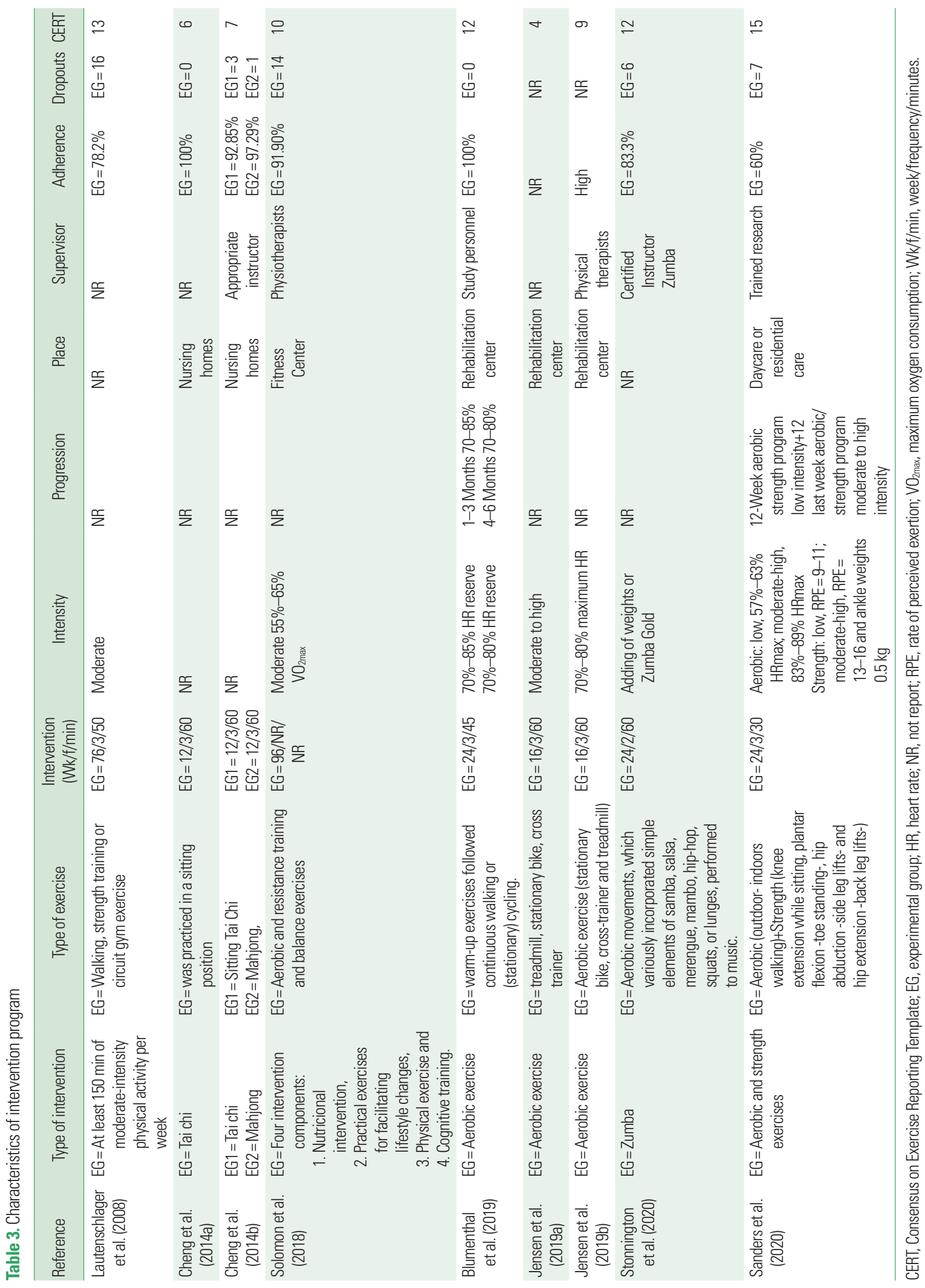




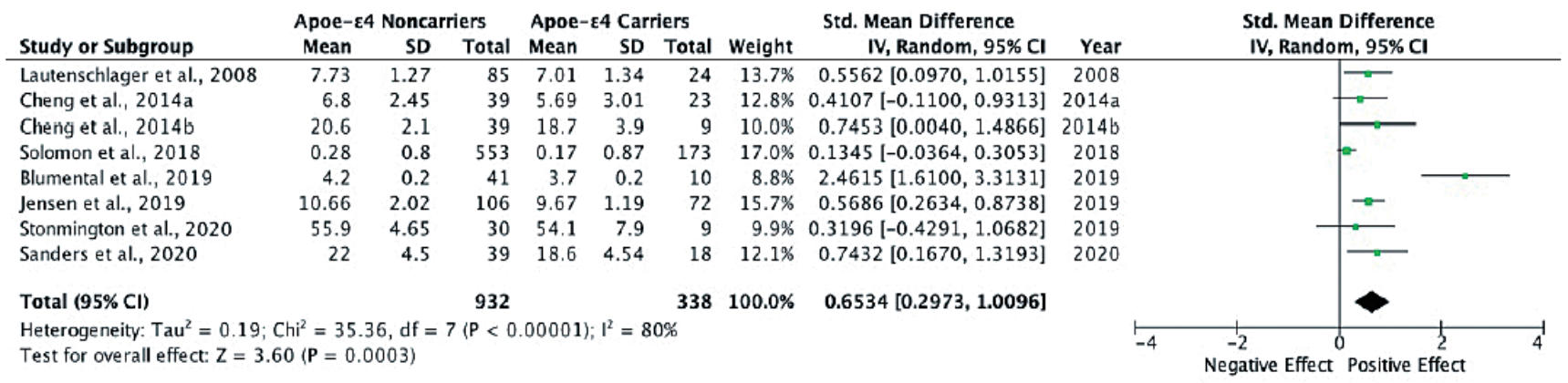

Intervention program at least 50 sessions

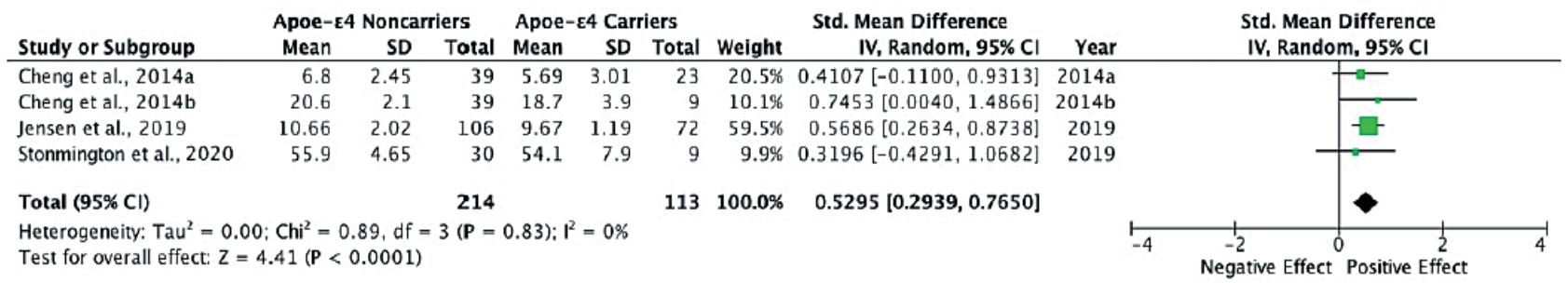

Intervention program more than $\mathbf{5 0}$ sessions

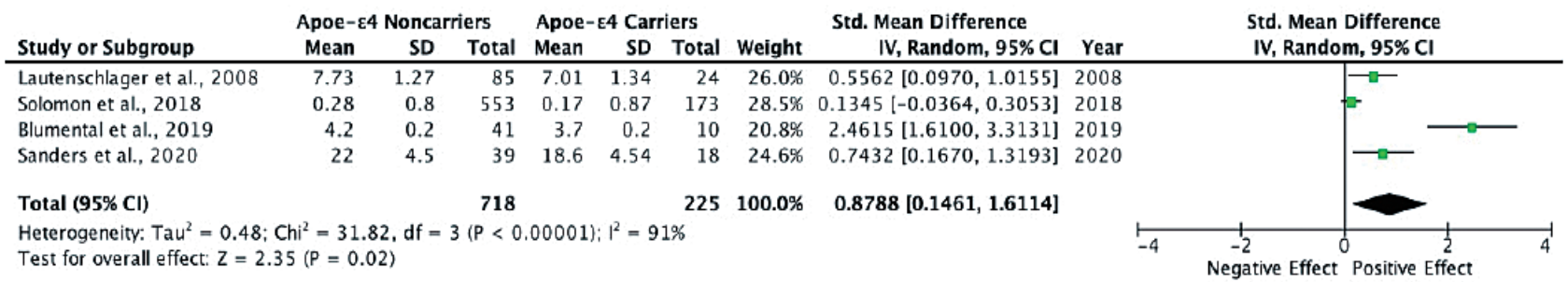

Low-Moderate intensity

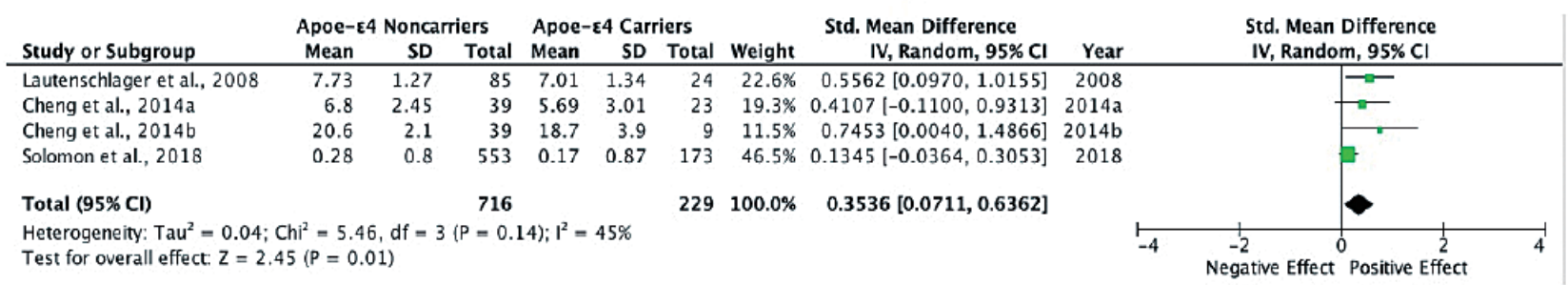

High Intensity

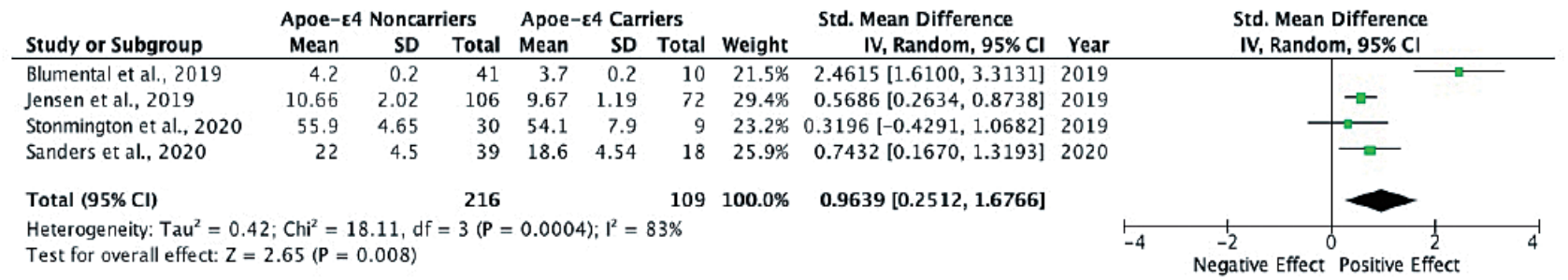

Fig. 2. Effect of intervention programmes on cognitive function. SD, standard deviation; Cl, confidence interval; $d f$, degrees of freedom. 
mean intervention adherence was $78.94 \% \pm 27.46 \%$. A total of 47 subjects dropped out the intervention programmes, with the maximum number per a dropped-out programme being 16 (Lautenschlager et al., 2008) and the minimum of 0 (Blumenthal et al., 2019; Cheng et al., 2014a). The analysis of the description of the intervention programmes was carried out following the instructions of the Consensus on Exercise Reporting Template-CERT (Slade et al., 2016). The CERT results revealed a low description of the intervention programmes (9.77 \pm 3.59$)$, whit Sanders et al., (2020) program being the best describes.

\section{Meta-analysis}

The meta-analysis was carried out on eight trials, as one did not report enough quantitative cognitive performance information (Jensen et al., 2019a). Fig. 2 shows the effect of intervention programmes on cognitive function in $\mathrm{ApoE} 4$ carriers and noncarriers. The results report that the effect of physical exercise on cognitive performance was greater in older adults' ApoE4 noncarriers than in ApoE4 carriers (Blumenthal et al., 2019; Cheng et al., 2014a; Cheng et al., 2014b; Jensen et al., 2019b; Lautenschlager et al., 2008; Solomon et al., 2018; Stonnington et al., 2020), being this significant difference $\left(\mathrm{SMD}=0.653 ; 95 \% \mathrm{CI}, 0.29-1.00\right.$; $\mathrm{chi}^{2}=$ 35.36; degrees of freedom $\left.[d f]=7 ; P<0.0001 ; I^{2}=80 \%\right)$.

The results of cognitive performance in older adults (ApoE4 carriers vs. noncarriers) based on the total duration of the intervention programme (<50 vs. $>50$ sessions) are shows in Fig. 2. The results report that lower volume intervention program $(<50$ sessions) generates similar effects on both groups ( $\mathrm{SMD}=0.529$; 95\% CI, 0.29-0.76; $\left.\mathrm{chi}^{2}=0.89 ; d f=3 ; P<0.83 ; I^{2}=0 \%\right)$. Higher volume intervention programmes ( $<50$ sessions) have a greater effect on cognitive performance in noncarriers older adults' ApoE4, being this significant difference $(\mathrm{SMD}=0.878 ; 95 \% \mathrm{CI}$, 0.14-1.61; $\left.\mathrm{chi}^{2}=31.82 ; d f=3 ; P<0.00001 ; I^{2}=91 \%\right)$.

Finally, Fig. 2 analyses how the intensity of the intervention program influences cognitive perfa difference significant $(\mathrm{SMD}=$ $0.963 ; 95 \%$ CI, $0.25-1.67 ; \mathrm{chi}^{2}=18.11 ; d f=3 ; P<0.0004 ; I^{2}=$ $83 \%)$.

\section{DISCUSSION}

The objective of this systematic review and meta-analysis was to study the role of physical exercise as a potentially preventive intervention in cognitive decline in older adults' ApoE4 carries and noncarriers, evaluating the quality of available evidence. It also aimed to investigate what type of physical exercise, how much (not weeks of intervention) and how intense this physical exercise generates a differential effect on cognitive performance in older adults' ApoE4 carries and noncarriers. There are a growing number of studies suggesting that physical exercise has positive effects on the cognitive functions of older adults (Du et al., 2018; Jia et al., 2019; Yu et al., 2006) as well as in older adults' carries ApoE4 (Smith et al., 2011; Smith et al., 2013; Woodard et al., 2012) reducing the risk of cognitive decline compared to sedentary people. The findings of this systematic review confirm that physical exercise contributes to positive effects on the cognitive functions of older adults by following the line of other studies (Rovio et al., 2005; Stephen et al., 2017) where physical exercise helps prevent and/or delay $\mathrm{AD}$, cognitive decline and other types of dementia, even in people carrying the ApoE4 allele as we can see in this study.

Aerobic and multimodal physical exercise is known to be beneficial for the cognitive functions of older adults (Lam et al., 2018; Lautenschlager et al., 2019). However, few studies have analysed the effect of other physical exercise modalities (strength, stretching, Pilates, aquatic exercise) on the cognitive function of older adults with ApoE4 carriers and noncarriers. In our systematic review, there are only two studies that combine aerobic and strength exercise (Sanders et al., 2020; Solomon et al., 2018). These studies indicated that performing this type of physical exercise generates positive effects on cognition, so we should continue to research in this line.

Despite increased evidence regarding the positive benefits of exercise in the health and functionality of older people with cognitive disorders, the specificities of prescription parameters are still little known. World Health Organization (2013) recommends physical exercise as a lifestyle to improve health in older adults, with improvements in cognitive function and positive neurophysiological changes. World Health Organization (2013) advises a minimum of 150 min per week of moderate-intensity aerobic exercise or 75 min of vigorous intensity with additional strengthening exercises. Of the nine studies analyzed, eight of them meet these recommendations, where one of them combines aerobic exercise with resistance training (Solomon et al., 2018). Barnard et al. (2014) states that adopting $40 \mathrm{~min}$ of fast walking 3 times a week as routine physical exercise can improve cognitive function. However, in our study we can observe that performing $30 \mathrm{~min}$ 3 times per week of physical exercise can also improve the cognitive functions of the older adult (Sanders et al., 2020) this follows the line of other studies (Jia et al., 2019) where it is stated that performing physical exercise up to 30 min per week improves the 
cognitive condition of patients. Based on our results, we could say that older people who do not carry out ApoE4 should perform 150 min per week of physical exercise, with a minimum of 50 sessions in order to perceive positive benefits over cognitive functions. With regard to the intensity of physical exercise, our study revealed that low-moderate intensity has the same effect on cognitive function in both groups (ApoE4 carriers and noncarriers), however at higher intensity of physical exercise, the effects have significant differences in cognitive performance in favour of noncarrier ApoE4.

Although methodological differences in exercise protocol (intensity, volume) make it difficult to compare samples, the studies analyzed indicate that aerobic interventions with a volume $>50$ sessions and a high intensity, are suitable for older adults carrying ApoE4 and ApoE4 noncarriers, with greater effects on noncarrier ApoE4. The primary strength of our meta-analysis lies in its multiple-subgroup design, which provides better quantification of the associations between specified amounts of physical exercise and relative effects on cognitive performance (ApoE4 carries and noncarries ApoE4). Another strength is that only RCT studies were included. The high sample size used in this analysis brings strength to the results of our study.

There are several limitations that might affect the reporting of our results. First, the studies are based on an aerobic physical exercise, therefore it cannot be determined whether the same effect can be achieved with other types of physical exercise. Second, there are very few studies to analyze. Another limitation of the study is not being able to know the duration of the effect after cessation of activity, because no study carried out a follow-up, so it will be very important that in future studies they track the behaviour of cognitive performance once for physical exercise stops. Due to the limitations set out above, more high-quality studies and RCT are needed to confirm the differential effect of physical exercise on cognitive performance in older adults' ApoE4 carriers/noncarriers.

\section{CONCLUSIONS}

Considering that there is no treatment to prevent the onset of cognitive decline in older adults' ApoE4 carriers and noncarriers, it is urgent to find the best way to delay the progression of the disease. Physical exercise is a low-cost, nonpharmacological therapy with no side effects that has been shown to help achieve this goal in a cost-effective and sustainable way. The results of this study show that aerobic physical exercise alone or combined with resistance training has a positive impact on cognitive functions of older adults whether or not they are carriers of ApoE4, with signifi- cant effects in favor of noncarrier ApoE4. Regarding the effect on cognitive performance that can generate different volumes and intensities of physical exercise programs, we should indicate that our results support volumes of more than 50 sessions (30-45 min) and high work intensities. However, due to the small number of studies analyzed, although high in the sample size used (2015 subjects), and the limitations presented above, we must present this data whit caution, so new RCTs are needed to contrast the results presented here.

\section{CONFLICT OF INTEREST}

No potential conflict of interest to this article was reported.

\section{ACKNOWLEDGMENTS}

The authors received no financial support for this article.

\section{REFERENCES}

Amieva H, Letenneur L, Dartigues J, Rouch-Leroyer I, Sourgen C, D'Alchée-Birée F, Dib M, Barberger-Gateau P, Orgogozo J, Fabrigoule C. Annual rate and predictors of conversion to dementia in subjects presenting mild cognitive impairment criteria defined according to a population-based study. Dement Geriatr Cogn Disord 2004;18:87-93.

Barnard ND, Bush AI, Ceccarelli A, Cooper J, de Jager CA, Erickson KI, Fraser G, Kesler S, Levin SM, Lucey B, Morris MC, Squitti R. Dietary and lifestyle guidelines for the prevention of Alzheimer's disease. Neurobiol Aging 2014;35:S74-S78.

Blumenthal JA, Smith PJ, Mabe S, Hinderliter A, Lin P, Liao L, WelshBohmer KA, Browndyke JN, Kraus WE, Doraiswamy PM. Lifestyle and neurocognition in older adults with cognitive impairments: a randomized trial. Neurology 2019;92:e212-e223.

Cheng S, Chow PK, Song Y, Yu ECS, Chan ACM, Lee TMC, Lam JHM. Mental and physical activities delay cognitive decline in older persons with dementia. Am J Geriatr Psychiatry 2014a;22:63-74.

Cheng S, Chow PK, Song Y, Yu ECS, Lam JHM. Can leisure activities slow dementia progression in nursing home residents? A cluster-randomized controlled trial. Int Psychogeriatr 2014b;26:637-643.

Corbo RM, Scacchi R. Apolipoprotein E (APOE) allele distribution in the world. Is $A P O E^{*} 4$ a 'thrifty' allele? Ann Hum Genet 1999;63:301-310.

De Souto Barreto P, Demougeot L, Vellas B, Rolland Y. Exercise training for preventing dementia, mild cognitive impairment, and clinically meaningful cognitive decline: a systematic review and meta-analysis. J Gerontol A 2018;73:1504-1511. 
Du Z, Li Y, Li J, Zhou C, Li F, Yang X. Physical activity can improve cognition in patients with Alzheimer's disease: a systematic review and meta-analysis of randomized controlled trials. Clin Interv Aging 2018; 13:1593.

Erickson KI, Weinstein AM, Lopez OL. Physical activity, brain plasticity, and Alzheimer's disease. Arch Med Res 2012;43:615-621.

Huang Y, Mahley RW. Apolipoprotein E: structure and function in lipid metabolism, neurobiology, and Alzheimer's diseases. Neurobiol Dis 2014;72:3-12.

Huebbe P, Nebel A, Siegert S, Moehring J, Boesch-Saadatmandi C, Most E, Pallauf J, Egert S, Müller MJ, Schreiber S. APOE $\varepsilon 4$ is associated with higher vitamin $\mathrm{D}$ levels in targeted replacement mice and humans. FASEB J 2011;25:3262-3270.

Hugo J, Ganguli M. Dementia and cognitive impairment: epidemiology, diagnosis, and treatment. Clin Geriatr Med 2014;30:421-442.

Jensen CS, Bahl JM, Østergaard LB, Høgh P, Wermuth L, Heslegrave A, Zetterberg H, Heegaard NH, Hasselbalch SG, Simonsen AH. Exercise as a potential modulator of inflammation in patients with Alzheimer's disease measured in cerebrospinal fluid and plasma. Exp Gerontol 2019a;121:91-98.

Jensen CS, Simonsen AH, Siersma V, Beyer N, Frederiksen KS, Gottrup H, Hoffman K, Høgh P, Frikke-Schmidt R, Sobol NA. Patients with Alzheimer's disease who carry the APOE $\varepsilon 4$ allele benefit more from physical exercise. Alzheimers Dement 2019b;5:99-106.

Jia R, Liang J, Xu Y, Wang Y. Effects of physical activity and exercise on the cognitive function of patients with Alzheimer disease: a meta-analysis. BMC Geriatr 2019;19:1-14.

Jongsiriyanyong S, Limpawattana P. Mild cognitive impairment in clinical practice: a review article. Am J Alzheimers Dis Other Demen 2018; 33:500-507.

Lam FM, Huang MZ, Liao LR, Chung RC, Kwok TC, Pang MY. Physical exercise improves strength, balance, mobility, and endurance in people with cognitive impairment and dementia: a systematic review. J Physiother 2018;64:4-15.

Lautenschlager NT, Cox KL, Ellis KA. Physical activity for cognitive health: what advice can we give to older adults with subjective cognitive decline and mild cognitive impairment? Dialogues Clin Neurosci 2019; 21:61.

Lautenschlager NT, Cox KL, Flicker L, Foster JK, Van Bockxmeer FM, Xiao J, Greenop KR, Almeida OP. Effect of physical activity on cognitive function in older adults at risk for Alzheimer disease: a randomized trial. JAMA 2008;300:1027-1037.

Mahley RW, Huang Y. Apolipoprotein e sets the stage: response to injury triggers neuropathology. Neuron 2012;76:871-885.

Michaelson DM. APOE $\varepsilon 4$ : the most prevalent yet understudied risk fac- tor for Alzheimer's disease. Alzheimers Dement 2014;10:861-868.

Moher D, Liberati A, Tetzlaff J, Tetzlaff J, Altman D, Antes G, Atkins D, PRISMA Group. Preferred reporting items for systematic reviews and meta-analyses: the PRISMA statement. PLoS Med 2009;6:1e00097.

Qian J, Wolters FJ, Beiser A, Haan M, Ikram MA, Karlawish J, Langbaum JB, Neuhaus JM, Reiman EM, Roberts JS. APOE-related risk of mild cognitive impairment and dementia for prevention trials: an analysis of four cohorts. PLoS Med 2017;14:e1002254.

Rajmohan R, Reddy PH. Amyloid-beta and phosphorylated tau accumulations cause abnormalities at synapses of Alzheimer's disease neurons. J Alzheimers Dis 2017;57:975-999.

Rawle MJ, Davis D, Bendayan R, Wong A, Kuh D, Richards M. Apolipoprotein-E (Apoe) $\varepsilon 4$ and cognitive decline over the adult life course. Transl Psychiatry 2018;8:1-8.

Rovio S, Kåreholt I, Helkala E, Viitanen M, Winblad B, Tuomilehto J, Soininen $\mathrm{H}$, Nissinen A, Kivipelto M. Leisure-time physical activity at midlife and the risk of dementia and Alzheimer's disease. Lancet Neurol 2005;4:705-711.

Sanders L, Hortobágyi T, Karssemeijer E, Van der Zee EA, Scherder E, Van Heuvelen M. Effects of low-and high-intensity physical exercise on physical and cognitive function in older persons with dementia: a randomized controlled trial. Alzheimer Res Ther 2020;12:1-15.

Schuit A, Feskens E, Launer L, Kromhout D. Physical activity and cognitive decline, the role of the apolipoprotein e4 allele. Med Sci Sports Exerc 2001;33:772-777.

Slade SC, Dionne CE, Underwood M, Buchbinder R. Consensus on exercise reporting template (CERT): explanation and elaboration statement. Br J Sports Med 2016;50:1428-1437.

Smith JC, Nielson KA, Woodard JL, Seidenberg M, Durgerian S, Antuono P, Butts AM, Hantke NC, Lancaster MA, Rao SM. Interactive effects of physical activity and APOE- $\varepsilon 4$ on BOLD semantic memory activation in healthy elders. Neuroimage 2011;54:635-644.

Smith JC, Nielson KA, Woodard JL, Seidenberg M, Rao SM. Physical activity and brain function in older adults at increased risk for Alzheimer's disease. Brain Sci 2013;3:54-83.

Solomon A, Turunen H, Ngandu T, Peltonen M, Levälahti E, Helisalmi S, Antikainen R, Bäckman L, Hänninen T, Jula A, Laatikainen T, Lehtisalo J, Lindström J, Paajanen T, Pajala S, Stigsdotter-Neely A, Strandberg T, Tuomilehto J, Soininen H, Kivipelto M. Effect of the apolipoprotein E genotype on cognitive change during a multidomain lifestyle intervention: a subgroup analysis of a randomized clinical trial. JAMA Neurol 2018;75:462-470.

Stephen R, Hongisto K, Solomon A, Lönnroos E. Physical activity and Alzheimer's disease: a systematic review. J Gerontol A Biol Sci Med Sci 2017;72:733-739. 
Stonnington CM, Krell-Roesch J, Locke D, Hentz JG, Dueck AC, Geda YE, Tariot PN, Caselli RJ. Impact of Zumba on cognition and quality of life is independent of APOE- $\varepsilon 4$ carrier status in cognitively unimpaired older women: a 6-month randomized controlled pilot study. Am J Alzheimers Dis Other Demen 2020;35:1533317519868370.

Tai LM, Thomas R, Marottoli FM, Koster KP, Kanekiyo T, Morris AW, Bu G. The role of APOE in cerebrovascular dysfunction. Acta Neuropathol 2016;131:709-723.

United Nations. Department of Economic and Social Affairs Population Division. World population ageing 2015 (ST/ESA/SERA/390). New York: United Nations; 2015.
Woodard JL, Sugarman MA, Nielson KA, Smith JC, Seidenberg M, Durgerian S, Butts A, Hantke N, Lancaster M, Matthews MA. Lifestyle and genetic contributions to cognitive decline and hippocampal structure and function in healthy aging. Curr Alzheimer Res 2012;9:436-446.

World Health Organization. Demencia, una prioridad de Salud Pública. Geneva (Switzerland): World Health Organization; 2013.

Yu F, Kolanowski AM, Strumpf NE, Eslinger PJ. Improving cognition and function through exercise intervention in Alzheimer's disease. J Nurs Scholarsh 2006;38:358-365.

Yu J, Tan L, Hardy J. Apolipoprotein E in Alzheimer's disease: an update. Annu Res Neurosci 2014;37:79-100. 